\title{
Mapa Falante de Crianças com Necessidades Especiais na Voz de Mães Cuidadoras
}

\author{
Andressa da Silveira, ${ }^{1}$ Bruna Pillar Benites Nicorena ${ }^{2}$
}

\begin{abstract}
RESUMO
Objetivo: Descrever a rede de apoio institucional acessada por crianças com necessidades especiais de saúde na voz de mães cuidadoras. Método: Pesquisa de abordagem qualitativa, desenvolvida com cinco mães cuidadoras de crianças com necessidades especiais de saúde, usuárias de uma Policlínica Infantil. O estudo foi desenvolvido por meio da dinâmica de criatividade e sensibilidade Mapa Falante, com a metáfora do desenho para a produção dos dados, realizada no espaço domiciliar de cinco crianças. As falas das mães cuidadoras foram audiogravadas, transcritas e analisadas por meio da análise de discurso pecheutiana. Resultados: Na perspectiva das cuidadoras, as crianças que possuem necessidades especiais de saúde carecem de uma ampla rede de apoio social, representada pelas redes institucionais e sociais, que fazem parte do dia a dia de seus filhos. No discurso dessas mulheres, observou-se a dificuldade de inclusão das crianças, a presença dos tabus sociais da necessidade especial de cuidados à saúde. As mães são cuidadoras principais; os cuidados são compartilhados em alguns momentos com os pais e avós. Conclusões: O cuidado solitário, algumas vezes compartilhado, é evidenciado no discurso das mães cuidadoras. A dificuldade de inserção da criança na escola e a diversidade de serviços de saúde requeridos, também aparecem na produção artística e nas enunciações dessas mulheres. O discurso denota a importância dos cuidados de enfermagem para o preparo da família que vai cuidar da criança no espaço domiciliar.
\end{abstract}

Palavras-chave: Saúde da criança. Necessidades e demandas de serviços de saúde. Apoio social. Relações mãe-filho.

SPEAKING MAP OF CHILDREN WITH SPECIAL NEEDS IN THE VOICE OF MOTHERS CAREGIVERS

\section{ABSTRACT}

Objective: to describe the institutional support network accessed by children with special health needs in the voice of caregiving mothers. Method: research with a qualitative approach, developed with mothers caring for children with special health needs, users of a Children's Polyclinic located on the western border of Brazil. The study was developed through the creativity and sensitivity dynamics Mapa Falante, with the metaphor of drawing for the production of data. The production of the data was carried out in the home space of five children, the speeches of the caregiving mothers were audio recorded, transcribed and analyzed through the analysis of the Pecheutian discourse. Results: from the perspective of caregivers, children who have special health needs need a wide network of social support, represented by institutional and social networks, which are part of their children's daily lives. In the speech of these women, it was also observed the difficulty of including children in society, due to the presence of social taboos of the special need for health care. Mothers are primary caregivers, care is sometimes shared with fathers and grandparents. Conclusions: solitary care, sometimes shared, is evidenced in the speech of caregiving mothers. The difficulty of inserting the child in school, the diversity of health services required also appear in the artistic production and in the statements of these women. The speech denotes the importance of nursing care for the preparation of the family that will take care of the child in the home.

Keywords: Child health. Health services needs and demand. Social support. Mother-Child relations.

\footnotetext{
Autora correspondente. Universidade Federal de Santa Maria, Campus Palmeira das Missões. Palmeira das Missões/RS, Brasil. https://orcid.org/0000-00024182-4714. andressadasilveira@gmail.com

2 Senac Uruguaiana. Uruguaiana/RS, Brasil.
} 


\section{INTRODUÇÃO}

A progressão científica e tecnológica na área pediátrica refletiu na sobrevida de crianças com necessidades de cuidados especiais de saúde, que receberam a denominação "children with special health care needs" (CSHCN) (MCPHERSON et al., 1998). No Brasil, essas crianças foram chamadas "crianças com necessidades especiais de saúde" (Crianes) (DOCHERTY et al., 2014).

Essas crianças possuem diversos diagnósticos e recebem uma classificação de acordo com as suas demandas de cuidados (NEVES et al., 2019), por meio de um instrumento de triagem que inclui as necessidades de cuidados requeridos: uso de medicamentos contínuos, utilização de serviços médicos, de saúde mental ou psicopedagógicos, limitação funcional, necessidade de reabilitação e tratamento/aconselhamento emocional, de desenvolvimento ou comportamento (BETHELL et al., 2008).

As Crianes representam uma população consumidora de cuidados de saúde, e cuidá-las exige habilidades diferenciadas de seus cuidadores e diversas fontes de apoio (BETHELL et al., 2008). Os cuidados contínuos e especializados das Crianes e as hospitalizações frequentes e prolongadas, requerem uma readequação familiar, considerando que no pós-alta hospitalar quem realiza os cuidados são os membros família no domicílio (SILVA et al., 2015).

Neste sentido, é fundamental que a rede de cuidados às crianças com algum tipo de deficiência seja articulada, com parceria entre os serviços de saúde que compõem a rede institucional, para que as necessidades de saúde sejam efetivamente atendidas (DUBOW; GARCIA; KRUG, 2018).

A rede institucional da Crianes inclui, também, pessoas que oferecem suporte no cotidiano, como a rede de cuidados complexos e os profissionais de saúde nela envolvidos. As redes de cuidados requeridas por Crianes são compostas pelos cuidadores, familiares e, sobretudo, por mães cuidadoras que lutam pelos direitos da criança de forma solitária. Desta forma, é primordial que a enfermagem e a equipe de saúde conheçam o cotidiano da Crianes e sua família, e que ambas sejam acompanhadas no pós-alta hospitalar para a articulação do cuidado em rede e minimizar as internações hospitalares (NEVES et al., 2015).

As redes de apoio social são a soma das relações entre o indivíduo e os componentes de uma sociedade, capazes de reduzir o isolamento social. Essa rede é um círculo social constituído por laços de afinidade formando uma espécie de teia que une as pes- soas (SILVEIRA; NEVES, 2012a). Essas redes construídas entre as famílias e as instituições de saúde devem ser estáveis e ativas, exercendo influência positiva na qualidade de vida e no estado de saúde dos usuários (NEVES; CABRAL; SILVEIRA, 2013).

$O$ presente artigo tem por objetivo descrever a rede de apoio institucional acessada por crianças com necessidades especiais de saúde na voz de mães cuidadoras. A partir dessas assertivas, apresenta-se, como questão do estudo: De que forma é constituída a rede de apoio institucional de crianças com necessidades especiais de saúde na perspectiva de mães cuidadoras?

\section{MÉTODO}

Este artigo apresenta resultados finais do relatório de um Projeto Matricial, com o desenvolvimento de cinco encontros individuais mediados pela Dinâmica de Criatividade e Sensibilidade (DCS) Mapa Falante no espaço domiciliar. As DCSs caracterizam-se como espaços de discussão e reflexão, levando os participantes da pesquisa a problematizarem suas vivências a partir da construção de uma produção artística, individual ou coletiva (CABRAL, 1998).

Trata-se de um estudo de abordagem qualitativa e participativa, a partir do Método Criativo e Sensível (MCS), utilizando a DCS Mapa Falante no intuito de representar, com a metáfora do desenho, as redes institucionais acessadas pelas Crianes.

A pesquisa foi desenvolvida com cinco mães cuidadoras de Crianes. A seleção das participantes ocorreu no decorrer das atividades práticas em uma Policlínica Infantil de referência, por meio da leitura de prontuários e a indicação da equipe de enfermagem sobre as crianças que apresentavam demandas de cuidados mistos (associação de dois ou mais cuidados de saúde).

Foram localizadas oito Crianes com demandas de cuidados mistos, e numeradas de um a oito para sorteio aleatório e ordem de convite para a produção dos dados. Desta forma, foram contatadas, inicialmente, duas famílias de Crianes, por meio de contato telefônico. Posteriormente, foram agendadas mais três visitas com outras mães, para a saturação de dados, totalizando cinco encontros/visitas.

Para a realização das DCSs utilizou-se cartolinas, folhas de ofício, canetas coloridas, lápis de cor e giz de cera. A dinâmica teve como questão geradora: "Quais os locais, serviços e instituições que seu filho(a) frequenta ou faz acompanhamento de saúde?" 
As produções artísticas foram fotografadas, e suas falas gravadas em mídia digital, transcritas e analisadas por meio da análise de discurso pecheutiana. Para sua operacionalização, utilizou-se a materialidade linguística e as ferramentas analíticas, como a metáfora, a paráfrase, a polissemia e o interdiscurso (ORLANDI, 2012).

Este artigo apresenta resultados de um Projeto Matricial, com resultados qualitativos, obtidos por meio do MCS com cinco mães/cuidadoras de Crianes. $O$ estudo seguiu os preceitos éticos e legais, obteve aprovação pelo Comitê de Ética e Pesquisa sob o número de CAAE 25453313.0.0000.5323 e parecer 498.734. Para manter o sigilo das informações obtidas e o anonimato das participantes, utilizou-se a letra " $M$ " referente à mãe, seguida por número ordinal aleatório.

\section{RESULTADOS}

O estudo foi composto por cinco mães cuidadoras de Crianes. Quanto à escolaridade, uma possuía Ensino Superior completo, duas Ensino Superior incompleto e duas Ensino Médio completo. Em relação à faixa etária, quatro possuíam entre 20 a 30 anos e outra mãe entre 30 e 40 anos de idade. Sobre o estado civil, três mães eram casadas e declararam que a Crianes é deste casamento, e outras duas mães declaram ser solteiras e não manter relação afetiva com o pai da criança.

As mães deste estudo afirmaram uma diversidade de cuidados requeridos por seus filhos, apoiados por uma longa trajetória percorrida em diferentes serviços de saúde. Sobre as demandas de cuidados apresentadas por essas crianças, suas mães declaram a presença do cuidado misto, por meio do uso de medicamentos, bota ortopédica, aparelho auditivo, óculos de grau, gastrostomia e sonda vesical. Em relação ao gênero das Crianes, três são meninos (até três anos de idade) e duas meninas (entre dois a oito anos de idade).

O filho de M1 apresentava perda auditiva profunda bilateral, com uso de aparelho auditivo e hipotonia; o filho de M2 apresenta esquizencefalia e diabetes; o filho de M3 possuía deficiência de hormônio do crescimento, com uso de gastrostomia e sonda vesical; a filha de M4 tem pé torto congênito equinovaro bilateral com uso de bota ortopédica; e a filha de M5 apresenta retinopatia da prematuridade com o uso de óculos.
A respeito das redes de apoio institucionais utilizadas pelas Crianes, as Figuras 1, 2, 3, 4 e 5 apresentam o Mapa Falante produzido no âmbito domiciliar e individualmente pelas cinco mães cuidadoras no momento da produção dos dados.

Figura 1 - Mapa Falante do filho de M1

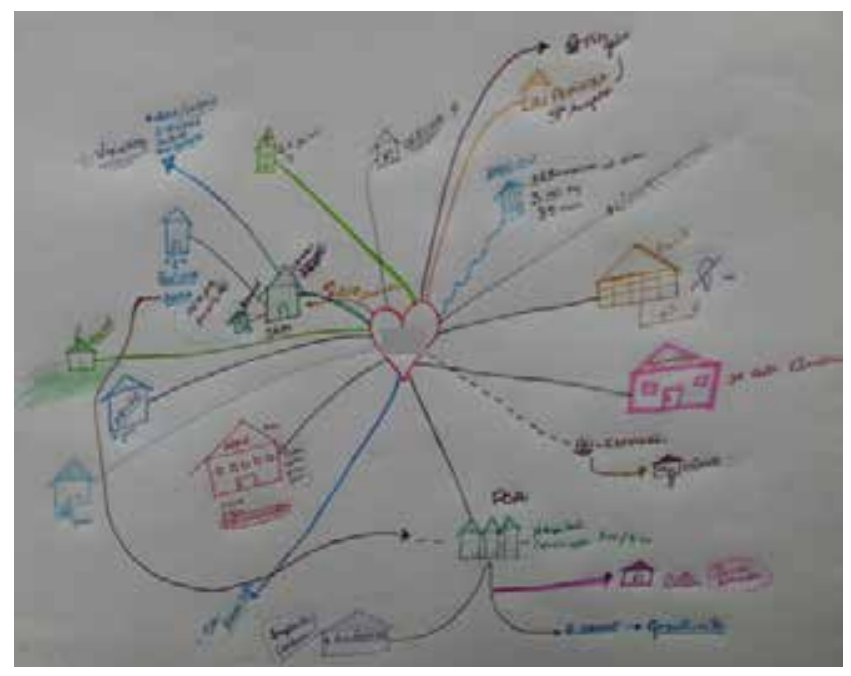

Figura 2 - Mapa Falante do filho de M2

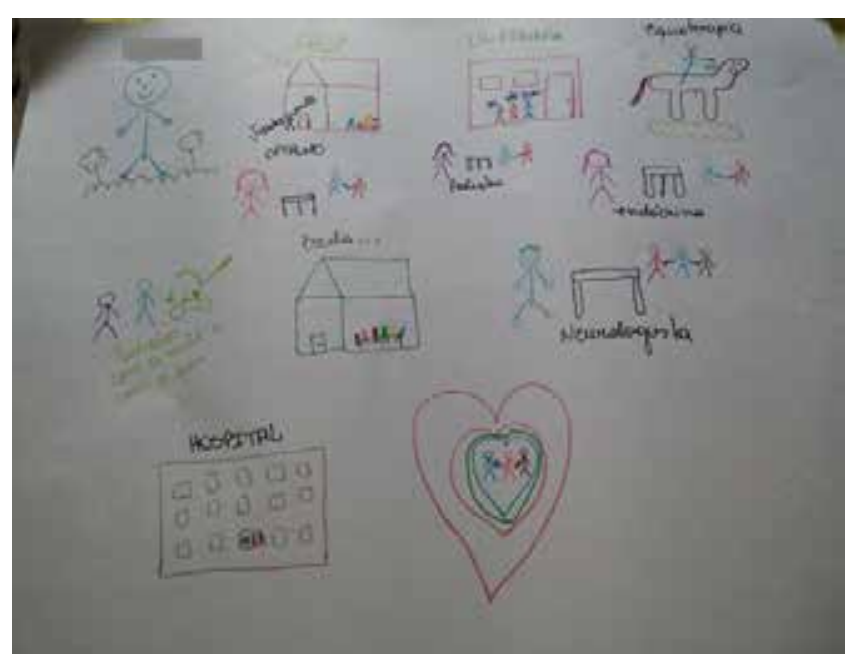

Figura 3 - Mapa Falante do filho de M3

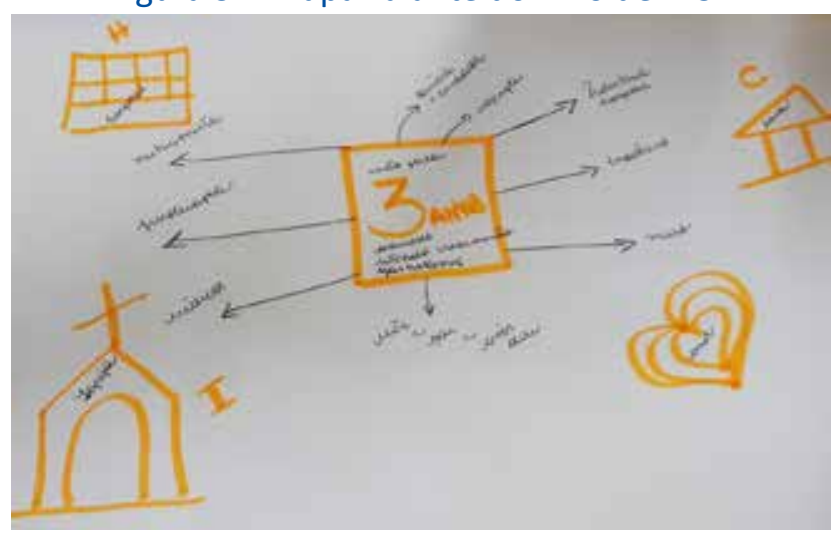


Figura 4 - Mapa Falante do filho de M4

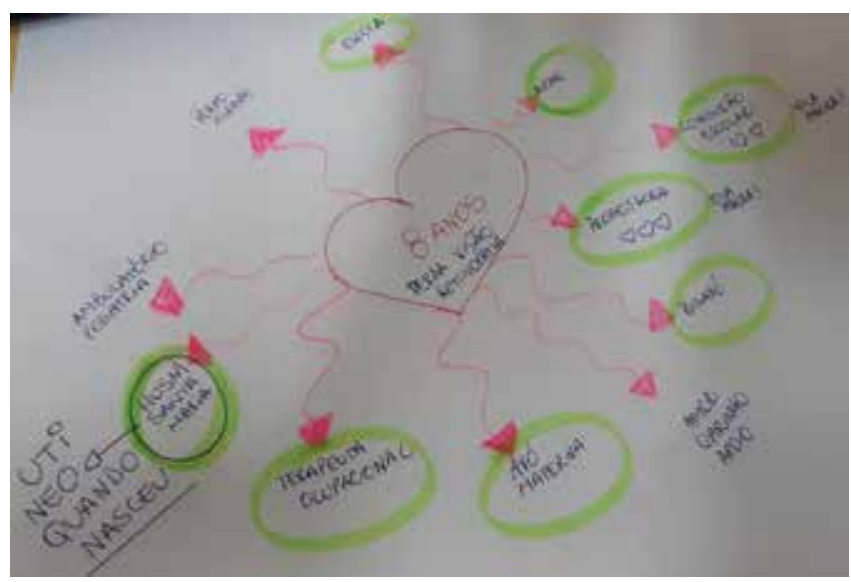

Figura 5 - Mapa Falante do filho de M5

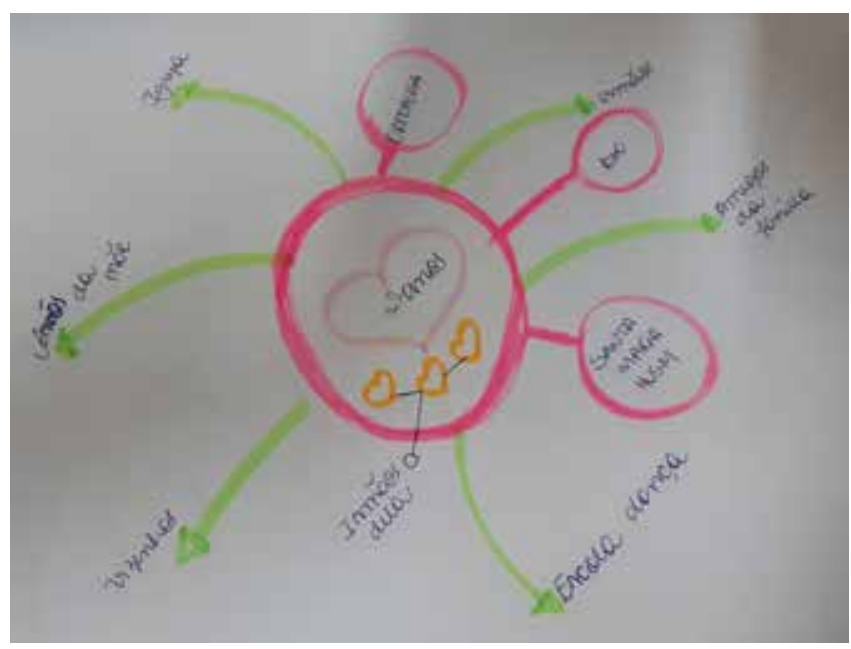

Entre as redes institucionais e profissionais de saúde, destacam-se: o Hospital, a Clínica da Associação de Pais e Amigos dos Excepcionais (Apae), a escola, a equoterapia, a fonoaudióloga, o pediatra, a fisioterapeuta, o neurologista, o otorrinolaringologista, o endocrinologista, a terapeuta ocupacional, o ortopedista, o oftalmologista, o Hospital Universitário, a Policlínica e a igreja.

Sobre a rede social e de convivência dessas Crianes, observa-se a presença das mães no desenvolvimento dos cuidados, em alguns momentos compartilhado com o pai da criança e avós maternas. Isso evidencia a presença de um cuidado materno e configurado nas mulheres da família da Crianes, na sobrecarga das mulheres e no cuidado essencialmente feminino. As enunciações das mães participantes do estudo apresenta a descrição das redes institucionais acessadas pelas Crianes:
Ele foi encaminhado para Porto Alegre; todo mês a gente vai no Hospital... [...] Ele frequenta a Apae, vai na fonoaudióloga, vai na fisioterapia... (M1)

Ele faz fisioterapia, faz equoterapia porque ele tem um déficit motor... [...] (M2)

Ele fica hospitalizado na Pediatria, por que tem a gastrostomia e a sonda... (M3)

Ela vai na escola, na Apae, usa o transporte, a condução que ela adora! (M4)

Tem a escola, tem a dança, ela vem pra cá... tem os manos que ela adora! (M5)

As mães destacaram o apoio recebido pelas avós das crianças e a importância de a Crianes frequentar a escola e a igreja como redes de apoio emocional.

[...] Tem a avó, a fisio, a fono e a Apae... E tem a igreja! (M1)

[...] Tem a avó! E ele gosta de ir na escolinha! (M2)

Eu vou na igreja, eu e o pai dele, ele é difícil eu conseguir levar... mas eu levo! (M3)

Ah, ela adora sair... Ela vai com a gente! (M4)

O discurso das mães traz a descoberta da necessidade especial de saúde da criança, a realização do pré-natal e que não foram preparadas em nenhum momento para cuidar de uma Crianes. Foi por meio da observação do desenvolvimento da criança, contudo, que perceberam algo diferente.

O meu pré-natal foi tudo bem! O profissional que me acompanhou nunca falou nada... Ele tinha cinco meses e era como se fosse um bebê recém-nascido, ele não tinha força muscular! (M1)

Fiz pré-natal, nenhuma alteração! A gente foi descobrir que ele tinha algum problema quando ele tinha um mês... [...] E com quatro meses ele começou a fazer fisioterapia... [...] (M2)

Ah, foi um choque. E depois que eu soube do hormônio do crescimento, muito gente perguntou se era uma síndrome, assim... se ele é especial... (M3)

Nasceu antes, prematura, ficou com cegueira depois da UTI Neo... (M4)

O pé vimos na hora, daí a gente já sabia, já esperava! (M5) 
Polissemicamente, algumas mães participantes do estudo enfatizam sobre o diagnóstico tardio de seus filhos, e, a partir disso, buscaram auxílio.

Ele mexia pouco na gestação, o profissional que estava me acompanhando também deveria ter notado, né?! (M1)

O médico só descobriu o diabetes quando ele tinha um ano e meio! (M2)

As construções discursivas vieram repletas de emoção, traduzidas nas falas dessas mulheres/mães e cuidadoras. Elas trouxeram os cuidados prévios durante a gestação, a ausência do medo e os questionamentos sobre como é ser mãe de um filho com necessidade especial de saúde.

Eu me cuidei toda a gravidez! Não fumava, não bebia, não saía e me cuidei superbem! Não entendia por que Deus tinha deixado acontecer aquilo comigo... (M1)

[...] Não fiquei com medo; não dimensionava as dificuldades que a gente iria enfrentar... Foi bem complicado, e hoje em dia também, quando eu vejo a diferença dele em relação às crianças... (M2)

A gente não esperava, mas ela é um presente! Cada conquista e cada descoberta! (M4)

Ela faz tudo... acompanha os manos em tudo! Então, não tem limite pra ela! (M5)

Os desafios enfrentados para cuidar da criança em casa sem preparo, a dicotomia entre o filho real e o filho esperado, também está presente nas enunciações das mães de Crianes:

Eu não reagi bem! Na verdade eu fui a que pior reagiu... Eu não aceitava o porquê que aconteceu comigo! (M1)

[...] Eu sei que é horrivel a gente ficar comparando, mas é muito difícil tu não fazer a comparação dele com outras crianças... (M2)

Pra mim o desafio é sair com ele no ônibus, as pessoas ficam olhando assim... "ele é normal?" ele é normal, ele não tem hormônio do crescimento e usa a sonda e a gastrostomia. Eu acho assim, Deus deu esse filho para eu cuidar! (M3)

No que se refere às redes de apoio institucional e social, algumas mães relataram sentir medo de seus filhos não serem acolhidos na sociedade e das restrições dessas crianças em atividades comuns a outras crianças.
Mandar um filho surdo pra escola, era isso que eu pensava, entendeu?! E é bem complicado de deixar na creche, porque, como ele usa aparelho auditivo... (M1)

[...] Eu acho que as professoras não incluem ele em todas as atividades, sabe? Principalmente nas brincadeiras que exigem da parte motora dele... (M2)

O difícil é que ele precisa de mim pra tudo... Eu quero que ele seja um menino normal, fazer coisas e comer coisas que as crianças da idade dele gostam... eu queria! (M3)

Na perspectiva das mães cuidadoras que participaram deste estudo, embora a Crianes necessite de cuidados de manutenção da vida e de uma ampla rede institucional, em alguns casos existem dificuldades em incluir seus filhos no espaço social. As enunciações trazem os tabus no entorno da demanda de cuidado apresentada, na sociedade que julga a aparência e a necessidade de saúde como limitação.

\section{DISCUSSÃO}

A rede institucional de uma Crianes é ampla e diversificada, integrada por diversos profissionais da área da saúde e educação, além dos serviços de saúde que a criança e sua família acessam. Já a rede familial está pautada em quem desenvolve os cuidados à criança. A família tem sua dinâmica modificada, configurada para atender às demandas de cuidados da Crianes e cria conexões de suporte social na rede institucional, na comunidade e por meio do apoio espiritual, percorrendo diversos segmentos de saúde em busca do tratamento adequado (NEVES et al., 2015).

As Crianes demandam cuidados ampliados e uma rede institucional de saúde composta por equipe multiprofissional. Os profissionais envolvidos nos cuidados dessa clientela devem ser capazes de assegurar acesso aos cuidados e assistência integral, interdisciplinar e intersetorial, em prol da reabilitação e inclusão social (MACHADO et al., 2018).

Para o cuidado de Crianes na comunidade é fundamental o processo de construção das redes de cuidado e espaços para a inclusão da criança na sociedade. O familiar cuidador, bem como a mãe/cuidadora, devem estar seguros para o desenvolvimento do cuidado domiciliar. Com isso, a rede de serviços de saúde deve ser capaz de estabelecer vínculos e de acolher a criança e sua família (DIAS et al., 2019). 
O acesso aos serviços de saúde, que compõe a rede institucional da Crianes, também está alicerçado na rede social de apoio, representado pela presença de familiares, amigos e profissionais que fazem parte do cotidiano da Crianes (AOKI et al., 2017).

Salienta-se sobre a responsabilização pelo cuidado do usuário, sobretudo a partir do serviço de atenção primária, posto que, embora o hospital seja o serviço de saúde mais utilizado pelas Crianes, a atenção primária deve cumprir o seu papel como porta de entrada na recepção e continuidade dos cuidados de saúde em rede (AOKI et al., 2017). Destaca-se, ainda, a inexistência de orientação a essas mães para a continuidade do cuidado na comunidade. Nesse sentido, é essencial a cooperação, a articulação e o consenso sobre ações terapêuticas voltadas para as crianças que possuem algum tipo de deficiência (MACHADO et al., 2018).

As famílias de Crianes necessitam de suporte social, emocional e afetivo a fim de preservar a vida e para a manutenção dos cuidados (MORAES; CABRAL, 2012). O processo de cuidado de uma Crianes perpassa pela dimensão emocional, social e financeira. A constituição da rede de apoio social é capaz de tornar seus indivíduos empoderados (VIEGAS; SOUSA, 2015; GARANITO; CURY, 2016).

O papel da mãe no contexto familiar é o de detentora do cuidado integral, ou seja, aquela que não mede esforços para proporcionar o melhor aos filhos, especialmente quando este apresenta alguma deficiência. Nestes casos, as mães são as principais cuidadoras destas crianças, cuja relação é baseada na dependência de cuidados e na necessidade de seus filhos (MORAES; CABRAL, 2012).

Cuidar de uma criança com necessidades especiais de saúde gera implicações que iniciam na descoberta do diagnóstico da criança, revelando a necessidade do enfrentamento de um processo doloroso diante da situação não planejada (SILVEIRA; NEVES, 2012b).

No que se refere aos cuidados de Crianes, destaca-se o importante papel das mães cuidadoras, que tem sido sinalizadas na literatura científica como as principais responsáveis pela execução dos cuidados (OKIDO; ZAGO; LIMA, 2015; FERREIRA et al., 2015). As mães acabam assumindo o papel central no cuidado em prol das necessidades de seus filhos, além do envolvimento emocional com a criança (SILVA; MILAGAIA, 2018).
Cuidar de uma Crianes incorpora uma série de atividades, muitas desenvolvidas exclusivamente pelas mães cuidadoras, que, por vezes, sentem-se, inclusive, sobrecarregadas diante das funções de cuidados exclusivos. Neste sentido, é fundamental compartilhar o cuidado das Crianes no espaço domiciliar, a fim de que este seja mediado com outros membros da família (BARBOSA et al., 2016; ELIAS; MURPHY, 2012; REIS et al., 2017).

Diante do exposto, é impreterível atuar na orientação, na educação em saúde e preparo para a alta hospitalar, na comunicação efetiva e nas redes de cuidados articuladas entre serviços oferecidos na atenção básica e hospitalar, a fim da continuidade dos cuidados de saúde apresentadas pela Crianes e que esses também incluam a família no processo de cuidado.

\section{CONCLUSÕES}

O Mapa Falante das crianças com necessidades especiais apresentou a configuração institucional composta por serviços de saúde e profissionais de referência para as demandas de cuidado apresentadas por Crianes. Nos discursos das mães cuidadoras participantes, é apresentada a diversidade de serviços de saúde requeridos por meio do Mapa Falante. A produção artística dessas mulheres revelou, ainda, que elas não foram preparadas para cuidar de seus filhos com necessidades especiais, contudo essas mães cuidam com o saber da experiência feita pelo exercício repetitivo do cuidado.

Quanto aos aspectos sociais, também mencionados nos discursos das mães cuidadoras participantes da pesquisa, existe o medo na inserção de seu filho na sociedade, configurado pela necessidade de cuidados; atividades comuns a outras crianças, como frequentar a escola, por exemplo, o receio se o filho está participando das brincadeiras propostas e as limitações para a socialização representadas pelo "não poder brincar e não poder comer".

O cuidado a essas crianças é configurado nas mulheres da família (mães e avós) e compartilhado com o pai. Existe, ainda, a dualidade entre o fiIho desejado e o filho real, posto que, embora essas mulheres tivessem realizado o pré-natal, algumas só souberam da necessidade especial de cuidado após o nascimento. $O$ estudo denota, também, a ocorrência da retinopatia vinculada à hospitalização em razão da prematuridade. 
Como limitações do estudo, destaca-se que as Crianes são procedentes de um serviço de saúde, não havendo comparação com outros cenários. Observou-se, ainda, que, embora haja diversidade de profissionais de referência para a Crianes, o profissional enfermeiro não foi enfatizado nas falas dessas mães.

Recomenda-se o desenvolvimento de pesquisas com abordagem participativa com famílias de Crianes. A utilização do Mapa Falante para a produção de dados mostrou-se favorável para temas sensíveis, traduzindo a emoção dessas cuidadoras.

Sugere-se atividades de ensino e extensão com o desenvolvimento de grupos de famílias cuidadoras na comunidade e que a assistência desenvolva práticas de educação em saúde e articulação da rede de cuidados e inserção social da Crianes.

\section{REFERÊNCIAS}

AOKI, M. et al. Desafios do cuidado em rede na percepção de preceptores de um Pet Redes em relação à pessoa com deficiência e bebês de risco: acesso, integralidade e comunicação. Cad. Bras. Ter. Ocup., v. 25, n. 3, p. 519-532, 2017. Disponível em: http://docs.bvsalud.org/biblioref/2018/02/879491/1813-8174-1-pb.pdf. Acesso em: abr. de 2020. ISSN 2526-8910.

BARBOSA, T. A. et al. Rede de apoio e apoio social às crianças com necessidades especiais de saúde. Rev. Rene., v. 17, n. 1, p. 60-6, 2016. Disponivel em: http://periodicos.ufc.br/ rene/article/view/2606/1994. Acesso em: abr. 2020.

BETHELL, C. D. et al. What is the prevalence of children with special health care needs? Toward an understanding of variations in findings and methods across three national surveys. Matern Child Health J., v. 12, n. 1, p. 1-14, 2008. Acesso em: abr. 2020.

CABRAL, I. E. O método criativo e sensível: alternativa de pesquisa na enfermagem. In: GAUTHIER, J. H. M. et al. Pesquisa em enfermagem: novas metodologias aplicadas. Rio de Janeiro: Guanabara Koogan; 1998. p. 177-203.

DIAS, B. C. et al. Desafios de cuidadores familiares de crianças com necessidades de cuidados múltiplos, complexos e contínuos em domicílio. Esc. Anna Nery, v. 23, n. 1, e20180127, 2019. Disponível em: http://www.scielo.br/scielo.php?script=sci_arttext\&pi$\mathrm{d}=\mathrm{S} 1414-81452019000100221 \& \mathrm{lng}=\mathrm{en} \& \mathrm{nrm}=\mathbf{i}$ iso. Acesso em: abr. 2020.

DOCHERTY, S. L. et al. A qualidade de vida de crianças que vivem com doenças crônicas ou complexas. In: HOCKENBERRY, M. J.; WILSON, D. (ed.). Wong: fundamentos de enfermagem pediátrica. 9. ed. Rio de Janeiro: Elsevier, 2014. p. 515-547.

DUBOW, C.; GARCIA, E. L.; KRUG, S. B. F. Percepções sobre a rede de cuidados à pessoa com deficiência em uma região de saúde. Saúde Debate, v. 42, n. 117, p. 455-467, 2018.
Disponível em: http://www.scielo.br/scielo.php?script=sci_arttext\&pid=S0103-11042018000200455\&Ing=en. Acesso em: abr. 2020.

ELIAS, E. R.; MURPHY, N. A. Council on Children with Disabilities. Home care of children and youth with complex health care 24 needs and technology dependencies. Pediatrics, v. 129, n. 5, p. 996-1005, 2012.

FERREIRA, M. C. et al. Avaliação do índice de sobrecarga de cuidadores primários de crianças com paralisia cerebral e sua relação com a qualidade de vida e aspectos socioeconômicos. Acta Fisiátr., v. 22, n. 1, p. 9-13, 2015.

GARANITO, M. P.; CURY, M. R. G. A espiritualidade na prática pediátrica. Rev. Bioét., v. 24, n. 1, p. 49-53, 2016. Disponível em: http://www.scielo.br/scielo.php?script=sci_arttext\&pid=S198380422016000100049\&lng=enh.

MACHADO, W. C. A. et al. Integralidade na rede de cuidados da pessoa com deficiência. Texto Contexto - Enferm., v. 27, n. 3, e4480016, 2018. Disponível em: http://www.scielo.br/ scielo.php?

MCPHERSON, M. G. et al. A new definition of children with special health care needs. Pediatrics, v. 102, n. 1, p. 137141, jul. 1998. Disponível em: http://pediatrics.aap publications.org/content/102/1/137.long. Acesso em: dez. 2019.

MORAES, J. R. M. M.; CABRAL, I. E. A rede social de crianças com necessidades especiais de saúde na (in) visibilidade do cuidado de enfermagem. Rev. Latino-Am. Enferm., v. 20, n. 2, 2012. Disponível em: http://www.scielo.br/scielo.php?pi$d=S 010411692012000200010 \&$ script=sci_arttext\&tIng=pt

NEVES, E. T. et al. Acesso de crianças com necessidades especiais de saúde à rede de atenção. Rev. Bras. Enferm., v. 72, supl. 3, p. 65-71, dez. 2019. Disponível em: http://www.scielo.br/scielo.php?script=sci_arttext\&pi$\mathrm{d}=\$ 003471672019000900065 \& \mathrm{lng}=\mathrm{en} \& \mathrm{nrm}=\mathrm{iso}$. Acesso em: 29 abr. 2020.

NEVES E. T.; CABRAL, I. E.; SILVEIRA, A. Rede familial de crianças com necessidades especiais de saúde: implicações para a enfermagem. Rev. Latino Am. Enferm., [Internet], 21(2), p. 562-570, 2013. Disponível em: http://www.scielo.br/scielo. php?script=sci_arttext\&pid=S010411692013000200562\&lng=en. Acesso em: 10 mar. 2015.

NEVES, E. T. et al. Rede de cuidados de crianças com necessidades especiais de saúde. Texto Contexto Enferm, v. 24, n. 2, p. 399-406, abr./jun. 2015. Disponível em: www.scielo. $\mathrm{br} / \mathrm{pdf} / \mathrm{tce} / \mathrm{v24n2/pt \_ 0104-0707-tce-24-02-00399.pdf.}$

OKIDO, A. C. C.; ZAGO, M. M. F.; LIMA, R. A. G. Care for technology dependent children and their relationship with the health care systems. Rev. Latino-Am. Enfermagem, v. 23, n. 2, p. 291-298, Apr. 2015. Disponível em: http://www.scielo.br/scielo.php?script=sci_arttext\&pi$\mathrm{d}=$ = 0104-11692015000200015\&lng=en\&nrm=iso. Acesso em: 29 abr. 2020.

ORLANDI, E. P. Análise de discurso: princípios e procedimentos. 10. ed. Campinas, SP: Pontes, 2012.

NOGUEIRA REIS, Kamilla Milione et al. A vivência da família no cuidado domiciliar à criança com necessidades especiais de saúde. Cienc. Enferm., v. 23, n. 1, p. 45-55, 2017. Dispo- 
nível em: https://scielo.conicyt.cl/scielo.php?script=sci_arttext\&pid=S0717-95532017000100045\&lng=pt\&nrm=iso. Acesso em: 29 abr. 2020.

SILVA, Franscisco Vaz da; MILAGAIA, Cristina. A inclusão na perspectiva de mães de crianças e jovens com necessidades especiais. Da Investigação às Práticas, v. 8, n. 1, p. 42-61, 2018. Disponível em: http://www.scielo.mec.pt/pdf/inp/ v8n1/v8n1a04.pdf.

SILVA, R. A. R. et al. Crianças com necessidades especiais de saúde: impacto no cotidiano familiar. Revista de Pesquisa Cuidado é Fundamental On-line, v. 7, n. 3, 2015, p. 2.902-2.909. Disponível em: https://www.redalyc.org/ pdf/5057/505750947041.pdf.

SILVEIRA, A.; NEVES, E. T. Crianças com necessidades especiais em saúde: cuidado familiar na preservação da vida. Cienc. Cuid. Saúde, v. 11, n. 1, p. 74-80, 2012a. Disponível em: http://www.revenf.bvs.br/scielo.php?script=sci_arttext\&pid=S1677-38612012000100010.

SILVEIRA, A.; NEVES, E. T. Vulnerabilidade das crianças com necessidades especiais de saúde: implicações para a enfermagem. Rev. Gaúcha Enferm., 33(4), p. 172-180, 2012b. Disponível em: http://www.scielo.br/scielo.php?script=sci_ arttext\&pid=S198314472012000400022\&lng=en.

VIEGAS, C. G. C.; SOUSA F. G. M. Necessidades técnicas-instrumentais do cuidador de crianças e adolescentes com diabetes. Enferm. Foco, v. 6, n. 1/4, p. 62-66, 2015. Disponível em: http://revista.portalcofen.gov.br/index.php/enfermagem/article/view/579/261. 